[Vicino Oriente XIX (2015), pp. 297-310]

\title{
L’ARCHIVIO DI TAVOLETTE DEL COMPLESSO B-C-H SULL’ACROPOLI DI BÜYÜKKALE
}

\author{
Tommaso De Vincenzi - Sapienza Università di Roma
}

\begin{abstract}
Through the comparison of the furnishings of worship of building $C$ on Büyükkale with the paleoHittite temple of Inandiktepe, especially pitchers of the so-called toreutical form with beak-spout terminated hook, new elements are emerged which seem to confirm the thesis that sees in this building a sanctuary.
\end{abstract}

Keywords: Hittites; building C; toreutical beak-hook pitchers; İnandıktepe; Büyükkale

\section{INTRODUZIONE}

Lo studio qui presentato si propone di essere una relazione preliminare riguardante il progetto di ricerca sull'archivio di tavolette del complesso di età imperiale B-C-H sull'acropoli di Büyükkale. In questo contributo saranno presentati i risultati parziali emersi dall'analisi comparativa fra il deposito archeologico dell'edificio C e il corpus di materiali repertati nei locali del tempio paleo-ittita di İnandiktepe.

Le ragioni alla base di un confronto fra classi di oggetti contestualizzati in due complessi architettonici cronologicamente distanti fra loro, sebbene circoscritto ad una specifica varietà di ceramica e di tipi di arredi cultuali, non si fondano solo su un'identità stilistica ma innanzitutto funzionale in stretta connessione alle celebrazioni liturgiche nei due complessi.

Nei paragrafi seguenti si tratteranno le principali argomentazioni per cui già in passato l'impiego degli oggetti sopraccitati è stato proposto collegarsi ad eventi cerimoniali.

Lo studio di queste tematiche si articolerà secondo uno schema analitico comparativo sperimentale, mediante cui si tenterà di integrare gli elementi che indicano le correlazioni con gli arredi cultuali dei due edifici. Un'annotazione importante pertinente il criterio di esposizione dei dati archeologici è che questi saranno presentati solo come fattori a supporto delle tesi convenzionali, e non propedeutici a nuove interpretazioni. I risultati emersi da questo studio preliminare, infatti, non hanno ancora fornito dati su possibili nuove interpretazioni per il corpo di fabbrica C. È da sottolineare, tuttavia, che fra gli specifici reperti rinvenuti nei locali dei due edifici, è stato evidenziato un elemento di connessione, legato non ad uno ma a due cerimoniali differenti, da cui scaturiscono tematiche che non intendiamo approfondire in questa presentazione.

\section{EDIFICIO C}

L'edificio C fu portato alla luce durante le campagne di scavo dal 1933 al $1937^{1}$ nei quadrati Q-S/15-17 nel settore sud-occidentale della cittadella di Büyükkale. Questa struttura si lega a due ulteriori corpi di fabbrica denominati B (quadrati Q-S/13-14) e H (quadrati S-U/14-17). Il complesso formato dai tre edifici si sviluppa secondo una pianta trapezoidale irregolare (fig. 1). Benché architettonicamente esso risulti essere una fabbrica indipendente, la disposizione agglutinante intorno alla corte a pilastri (quadrati I-M/5-7) dei

$1 \quad$ Bittel 1935, 13-28; 1937, 1-26. 
fabbricati D (quadrati M-R/8-12) e A (quadrati T-W/6-11), comunicanti per il tramite del Verbindungsbau (quadrati R-T/12-13) alla Torbau (quadrati T-U/11-13), pare descrivere uno schema topografico, il cui vertice deve essere indicato nel cosiddetto palazzo reale $\mathrm{D}^{2}$. In base a questa ipotesi, pertanto, il complesso formato dai corpi di fabbrica B-C-H non apparirebbe più come una struttura indipendente, ma quale unità interdipendente di un coerente progetto architettonico ${ }^{3}$. La costruzione del complesso B-C-H si data alla seconda fase del progetto di monumentalizzazione della cittadella di Büyükkale, corrispondente a BK IIIb, avviato all'inizio del periodo Imperiale, BK IIIa, tra il XIV e il XIII secolo a.C. circa (tab. 1).

\begin{tabular}{|c|c|c|c|c|c|c|}
\hline Periodo & Quadrato & \multicolumn{4}{|c|}{ Costruzione edifici } & Inventario \\
\hline & & Descrizione & Fondamenta & Tipo edificio & Pavimento & \\
\hline IIIb & $\mathrm{R}-\mathrm{T} / 12-13$ & Edificio VB & & $\begin{array}{c}\text { Edificio di } \\
\text { collegamento } \\
\text { con piano } \\
\text { inferiore e } \\
\text { sopra } \\
\text { colonnato } \\
\end{array}$ & & $\begin{array}{c}4 \text { bacini in } \\
\text { argilla nel } \\
\text { vano } 2 \text { piano } \\
\text { inferiore }\end{array}$ \\
\hline IIIb & T-U/11-13 & Torbau I & $\begin{array}{c}\text { Opera in } \\
\text { muratura in } \\
\text { calcare blocchi } \\
\text { larghi } 75-100 \\
\text { cm }\end{array}$ & $\begin{array}{l}\text { Porta urbica } \\
\text { metà sud- } \\
\text { ovest edificio } \\
\text { a due piani }\end{array}$ & & \\
\hline IIIb & Q-S/13-14 & Edificio B & $\begin{array}{c}\text { Opera in } \\
\text { muratura in } \\
\text { blocchi di } \\
\text { calcare larghi } \\
75-100-130 \mathrm{~cm}\end{array}$ & $\begin{array}{c}\text { Edificio a } \\
\text { multi-vani su } \\
\text { due piani }\end{array}$ & $\begin{array}{c}\text { Argilla } \\
\text { battuta vani } \\
\text { piano } \\
\text { inferiore }\end{array}$ & $\begin{array}{l}24 \text { frammenti } \\
\text { tavolette nel } \\
\text { riempimento } \\
\text { vano } 5\end{array}$ \\
\hline IIIb & Q-S/15-17 & Edificio C & $\begin{array}{c}\text { Edificio } \\
\text { cultuale con } \\
\text { bacino }\end{array}$ & $\begin{array}{c}\text { Opera in } \\
\text { muratura con } \\
\text { grandi } \\
\text { blocchi } \\
\text { basamento } \\
\text { bacino } \\
\text { blocchi } \\
\text { ciclopici } \\
\text { larghi } 75 \text {, } \\
\text { 100, } 120,150 \\
\text { cm orlatura } \\
\text { vani } 4 \text { e } 6\end{array}$ & & $\begin{array}{l}\text { Numerosi } \\
\text { vasi votivi- } \\
\text { (lucerne), } \\
\text { conchiglie, } \\
\text { schegge di } \\
\text { ossidiana, } \\
\text { lame in pietra } \\
\text { nella sabbia } \\
\text { alluvionale } \\
\text { entro bacino } \\
2 \text { idoli in } \\
\text { argilla vano } 1 \\
\text { stele di } \\
\text { Tuthalija } \\
\text { vano fronte } \\
\text { vano } 2\end{array}$ \\
\hline IIIb & S-U/14-17 & Edificio $\mathrm{H}$ & $\begin{array}{l}\text { Edificio adibito } \\
\text { a magazzino a } \\
\text { due piani con } \\
\text { porticato a } \\
\text { pilastri frontale }\end{array}$ & $\begin{array}{l}\text { Alzato in } \\
\text { mattoni } \\
\text { parete } \\
\text { occidentale }\end{array}$ & & \\
\hline
\end{tabular}

Tab. 1 - Tabella stratigrafica ed inventario del complesso B-C-H (adattata da Neve 1982, tab. 9).

2 Bittel 1935, 17.

3 Cfr. nota 2. 
L'edificio C si compone di cinque stanze a pianta rettangolare (Nr. I-II, IV-VI) articolate intorno ad un ambiente centrale (III) quadrangolare di 5,20 x 6 m. L’ingresso si apriva sul prospetto orientale del vano II, che misura 7 × 4,50 m, da cui si accedeva, presumibilmente, ai vani laterali I (di 17,5 x 4,50 m) e V (di 9 x 4,50 m) ${ }^{4}$. Data la conservazione delle pareti a livello di fondazione, tuttavia, non si conosce come si sviluppasse la percorrenza all'interno dell'edificio che presenta due fasi di costruzione. Benché fra queste due fasi non risultino alterazioni della planimetria, nella tecnica di costruzione, come per il materiale impiegato, si osservano modifiche architettoniche rilevanti. Nella fattispecie, questi interventi strutturali posteriori appaiono essere stati circoscritti alle mura dell'ambiente centrale III. Diversamente dagli altri ambienti, le cui fondazioni erano state erette per mezzo di semplici blocchi in pietra con alzati in mattoni, l'ampiezza delle mura del vano III fu ispessita considerevolmente per la sovrapposizione, sulla sottostruttura precedente, di lastroni di calcare lunghi oltre i $2 \mathrm{~m}$, e con i prospetti esterni levigati (fig. 2). Parallelamente all'ampliamento dello spessore delle fondazioni il pavimento correlato alla prima fase di costruzione fu livellato da uno strato di riempimento, che funse da base per il posteriore piano di calpestio, sulla cui superficie furono repertati i tre esemplari di brocca per libagioni, che saranno analizzati dettagliatamente nei paragrafi successivi, insieme il restante corpus del deposito archeologico.

\subsection{Elementi interpretativi dell'edificio $C$ quale santuario}

Le suddette modifiche architettoniche relative al vano III, risalenti alla seconda fase di costruzione (BK IIIb), evidenziano per questo ambiente una funzione particolare, già dedotta da Kurt Bittel sulla base del materiale rinvenuto, che rappresenta la ragione per l'interpretazione di tale fabbrica quale Schrein ${ }^{5}$. Egli infatti istruì questa tesi in relazione al ritrovamento in situ delle centinaia di frammenti di vasi votivi (Votivegefasse) (fig. 3), ma anche esemplari integri, quali un tipo di brocca con ansa a nastro e corpo bi-conico con base convessa ed estremità a puntale ${ }^{6}$, semplici ciotole con base piatta oppure con piede ad anello $^{7}$, sostegni per vasi con profilo tronco-conico ${ }^{8}$, e ancora tipi di bottiglie, il primo, con corpo bi-conico, lungo collo rastremato con ansa ad arco e base ad anello, il secondo, corpo ovoidale, alto collo slanciato, base fortemente convessa con estremità ad anello ${ }^{9}$ e, specialmente, tre esemplari di una brocca con versatoio terminante ad uncino, ansa a gomito direttamente connessa al bordo, collo lungo e sottile, corpo bi-conico innestato su un basamento a calice con piede ad anello (fig. 4:c-e). K. Bittel ${ }^{10}$, e successivamente anche Franz Fischer ${ }^{11}$, escluse, per questa varietà di brocca, in rapporto allo spessore troppo sottile del supporto a calice, e alla limitata capacità volumetrica del corpo, un uso per

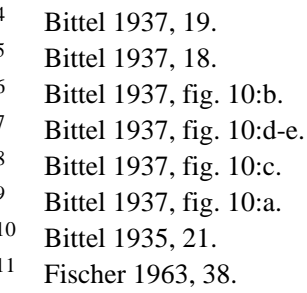


attività domestiche, e, per analogia con un'illustrazione della medesima, su un rilievo da Alaca Höyük, propose che essa venisse utilizzata unicamente quale vaso per libagioni ${ }^{12}$.

Nella citata scena a rilievo il re ittita è rappresentato nell'atto di procedere alla guida di una processione rituale, formata dalla regina e da due offerenti, verso una divinità seduta in trono (fig. 5). Il fulcro di questa scena è indicato nell'atto cerimoniale della libagione che il re effettua versando il liquido contenuto nel vaso direttamente ai piedi del dio. Il sovrano solleva il vaso per l'ansa con la mano sinistra, in posizione orizzontale, mentre con la destra stringe al petto il lituo con l'estremità ricurva. La base ad anello, come anche il corpo biconico, paiono essere gli elementi stilistici principali che identificano tale recipiente con la varietà di brocca repertata nel vano III dell'edificio C. Benché la scena illustrata sopra questo ortostato non possa essere associata con sicurezza a uno specifico rituale, appare evidente l'impiego di detta classe ceramica nei cerimoniali ittiti, come ipotizzato da $\mathrm{K}$. Bittel. Un'ulteriore conferma a tale ipotesi emerge in rapporto ad una seconda rappresentazione di questa brocca, parimenti a rilievo, sebbene realizzata attraverso una tecnica differente, quale quella dei vasi con fregi a rilievo. Dagli scavi di Karahöyük ${ }^{13}$, infatti, proviene un frammento di vaso con una scena che ritrae un uomo, presumibilmente partecipe di un cerimoniale religioso, che con la mano destra pare afferrare il collo di una brocca (fig. 6) stilisticamente paragonabile agli esemplari sopraccitati di Boğazköy e Alaca Höyük (fig. 4:f).

Quantunque il contesto stratigrafico dei tre esemplari del vano III dati tale classe ceramica all'epoca imperiale ittita, pare, tuttavia, complesso tracciarne il processo evolutivo stilistico, considerata la quantità di varianti, che $\mathrm{F}$. Fischer ha raccolto sotto la denominazione di forma toreutica (toreutische Form) ${ }^{14}$, e di cui i modelli da Boğazköy e Alaca Höyük ${ }^{15}$ rappresenterebbero un'evoluzione posteriore a latere. I tipi antesignani di tali brocche si identificano quasi sicuramente in tre esemplari rinvenuti nel quadrato $\mathrm{M} / 18$ a Büyükkale (nel cosiddetto Gefäßraumes) ${ }^{16}$ e nella Città Bassa quadrati $\mathrm{J} / 20 \mathrm{~h}-7 / \mathrm{d}^{17}$ e $\mathrm{J} / 20$ i-lc- $2 \mathrm{a}^{18}$, strato 4 , che in base all'analisi testuale delle tavolette paleo-assire sarebbe coevo con il livello Ib del kârum di Kanesh/Kültepe. Stilisticamente questi ultimi si differenziano per la fabbrica realizzata mediante un elevato livello tecnico che si riscontra nel trattamento della superficie esterna rivestita di una tonalità marrone lucido, che pare riprodurre il riflesso lucente del metallo. Si ritiene, infatti, che queste forme ceramiche fossero una copia di modelli in metallo e, pertanto, che l'effetto lucido fosse appositamente ricercato dal ceramista, per ricreare la tipica lucentezza dei vasi in rame ${ }^{19}$. La forma, parimenti, si sviluppa in una profonda vasca emisferica capovolta, decorata da una coppia di cuppelle, cui si allega un'ansa ad arco modellata sul collo lungo e affusolato con versatoio rivolto verso l'alto, innestata su di un basamento a calice con piede ad anello. Una variante

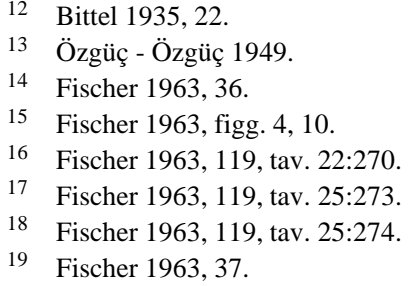


ulteriore, molto importante nell'ottica di tale studio, appare essere rappresentata, da alcuni frammenti con caratteristico versatoio terminante a uncino, collegata ad una base a calice fortemente rastremata verso l'alto. Benché stilisticamente si evince che quest'ultima brocca sia omologata alla cosiddetta toreutische Form, risulta, tuttavia, essere incerto se essa sia coeva alla fase finale del kârum, quindi precipua di questo periodo, o rappresenti una forma evoluta posteriore in epoca Antico Ittita. Tale problematica, infatti, risulta istruirsi nel merito della contestualizzazione stratigrafica dei frammenti di questa seconda variante, ritrovati a Büyükkale nel vano XI h edificio G livello $B K I V c^{20}$, e nella Città Bassa, quadrati J/20 h-7/d-2 strato $4^{21}$, J-K/20-21 e K/20 strato $2^{22}$.

Nella fattispecie la posizione stratigrafica in BK IVc di questi frammenti non risulterebbe essere un indicatore valido per la continuità di questa varietà di brocca anche per l'epoca paleoittita, poiché si ritiene che la contestualizzazione dei frammenti suddetti fosse correlata al processo di erosione, che ne provocò l'infiltrazione nei posteriori livelli insediamentali della Città Bassa e di Büyükkale ${ }^{23}$. Un elemento che conferma la continuità di questa forma di brocca cosiddetta "toreutica" nella sua versione con versatoio con terminazione a rampone e base a calice fortemente rastremata, nella fase post-Kanesh, quindi in epoca Antico Ittita, è la scoperta di due esemplari integri nei locali del tempio di İnandıktepe (fig. 4:a-b), la cui distruzione risale alla fine del XVI sec. a.C. ${ }^{24}$. I vasi furono rinvenuti nella stanza 2 che Tahsin Özgüç, parallelamente ai vani 1, 3, 4 (quadrati G-H-I/2$6)^{25}$, identificò nei depositi templari, a ragione del campionario ceramico rituale ${ }^{26}$. Dei due esemplari di İnandıktepe è la brocca nella versione con vasca emisferica capovolta che stilisticamente si conforma ai sopraccitati frammenti nel livello BK IV b a Büyükkale, e negli strati 2 e 4 della Città Bassa. La correlazione fra questa varietà antesignana di brocca toreutica con versatoio e gli esemplari del vano III nell'edificio C, tuttavia, non si stabilisce unicamente in ragione dell'analisi comparativa dello stile, bensì si articola specificatamente per il suo uso quale vaso per libagioni nei cerimoniali, che si evince dalle scene a rilievo rappresentate sull'ortostato da Alaca Höyük e sul vaso con fregi da İnandıktepe.

Sottostante lo strato di crollo nella camera 2, addossato alla parete nord, infatti, lo scavo riportò alla luce un vaso a quattro anse di grandi dimensioni ${ }^{27}$, dipinto con quattro fasce a rilievo applicate sulla superficie, e ripartite in registri mediante delle strisce orizzontali decorate con motivi geometrici (fig. 7:a). Le scene illustrate rappresentano una processione

20 Fischer 1963, 118; tav. 24:244-247.

21 Fischer 1963, 118; tav. 25:273.

22 Fischer 1963, 119; tav. 27:275-276, 280-281.

23 Fischer 1963, 37-38.

24 Özgüç 1988, 110-111.

25 Özgüç 1988, pianta 1.

26 Özgüç 1988, 81. Tale codifica si articolava in base alla varietà delle forme ceramiche rituali fra cui un tipo di calice a quattro anse con orlo piatto estroflesso e profonda vasca carenata, innestata su un basamento conoidale e base ad anello (Özgüç 1988, 81-82, figg. 20-22); un grande vaso con vasca biconica e protome taurina applicata al bordo, con base convessa e terminazione a puntale (Özgüç 1988, 79, fig. 28:a-b, tav. 25, 1a-c); e ancora una brocca del tipo cosiddetto lentoide, di cui si osservano due varianti: una con vasca globulare e grande ansa ad anello che si unisce all'alto collo cilindrico, e munita di due prese più piccole laterali (Özgüç 1988, 80, tav. 28:1a-b), e una seconda con vasca anch'essa globulare compressa al centro, rinvenute sul pavimento della stanza 2 (Özgüç 1988, 80, tav. 28:2a-b).

27 Altezza vaso $82 \mathrm{~cm}$, larghezza orlo $46 \mathrm{~cm}$, diametro corpo $43 \mathrm{~cm}$ (Özgüç 1988, 84). 
eterogenea, composta dal personale addetto al tempio con la compartecipazione di musicanti, danzatori, acrobati, che, si ritiene, festeggi il cerimoniale del matrimonio sacro fra le coppie di divinità Dupašiya-Inara o Telepinu-Hatepinu ${ }^{28}$. La scena pertinente l'ipotetica cerimonia nuziale è descritta nel terzo registro, sopra una piattaforma posta innanzi ad un edificio, riconosciuto come tempio consacrato al dio della Tempesta, per la presenza di una statua di toro stante sopra un altare, alla cui base viene immolato un secondo toro, nel registro della seconda fascia ${ }^{29}$.

T. Özgüç, in base lo studio degli elementi iconografici e dall'esame dei testi epigrafici rituali, ipotizzò che i rilievi applicati sul vaso di İnandıktepe rappresentassero un matrimonio rituale fra due divinità associato alla celebrazione del trentottesimo giorno della festa di primavera dell'AN.TAH.ŠUM che veniva celebrata in epoca imperiale, benché le sue origini risalirebbero al periodo Antico Ittita ${ }^{30}$.

Il legame fra la narrazione e la versione toreutica di brocca con versatoio si manifesta in riferimento alla scena libatoria rappresentata all'estremità sinistra del secondo registro del vaso. In essa, infatti, si nota una figura maschile sacerdotale (N. 33) in piedi fronte a un altare (N. 32) nell'atto di compiere la libagione con la mano destra che tiene sollevata la brocca e ne versa il liquido contenuto, in una ciotola/calice, offertagli dalla divinità stessa, seduta sopra una sedia pieghevole dietro l'altare (N. 31); la mano sinistra è levata in un gesto di adorazione (fig. 7:b-d). Sebbene risulti essere vincolante il parallelo iconografico fra le scene libatorie rappresentate rispettivamente sull'ortostato da Alaca Höyük e sul vaso di İnandıktepe, proprio per l'uso di tale varietà toreutica di brocca quale vaso per libagioni, tanto per l'epoca antico-ittita, quanto per l'età imperiale, la formula cerimoniale appare essere difforme, come si evince in relazione ai canoni illustrativi precipui il rituale di İnandıktepe e di Alaca Höyük. In riferimento al primo dei due esempi citati, infatti, il rito libatorio rappresentato all'estremità sinistra della seconda fascia, si distingue come un episodio correlato a margine della scena primaria, benché concomitante e propedeutico alla celebrazione del matrimonio sacro, effettuato da un sacerdote che si differenzia rispetto agli altri personaggi partecipi al corteo soltanto per gli attributi che porta e per l'azione compiuta. Nel secondo caso, invece, è proprio nel rito libatorio che si accentra il rituale, poiché è il re ittita stesso in testa a una processione che liba ad una divinità seduta in trono. Rispetto al vaso di İnandıktepe, pertanto, la rappresentazione religiosa nel rilievo di Alaca Höyük, per il tramite della figura del re, che officia in prima persona, specifica la liturgia fondante il culto stesso.

Benché, come sottolineato nel $\S 1$., allo stato attuale della ricerca non siano emersi dati utili ad identificare quale tipologia di culto fosse celebrata nell'edificio $\mathrm{C}$, del quale si è supposta un'interpretazione come santuario, da un'analisi comparativa con gli arredi cultuali portati alla luce nei locali del tempio a İnandıktepe si è dedotta una presunta correlazione fra i due corpi di fabbrica, senza che con questo si voglia alludere ad un’omologazione liturgica. Contestualmente a ciò, si citano i seguenti reperti, rinvenuti nella fossa circolare, profonda $10,40 \mathrm{~m}$ con un diametro di 5,50 m, scavata all'interno

28 Yildirım 2009, 243.

29 In riferimento alla visione prospettica dei rilievi la scena raffigurante il matrimonio sacro doveva, con tutta probabilità, svolgersi all’interno del tempio dedicato al dio della Tempesta.

30 Özgüç 1988, 126 
dell'ambiente 29 nel tempio di İnandıktepe, nei quadrati A-B-C/4, in una fase successiva alla distruzione dell'edificio ${ }^{31}$ : riproduzione in miniatura di un santuario a pianta rettangolare, chiuso in alto e sui lati, ma aperto sul davanti su una corte anteriore, al cui interno era ricoverata una figura fittile di divinità maschile seduta in trono della quale si riscontra un chiaro parallelo nell'esemplare trovato sul piano pavimentale del vano 1 nell'edificio C (fig. 8:a-b); e un'impugnatura in calcare con profilo a fungo, dalla superficie accuratamente levigata (fig. 9) ${ }^{32}$, con cui un paragone si fonda con la copia nel vano 5 , quadrato $\mathrm{R} / 14$, dell'edificio $\mathrm{B}^{33}$, rinvenuta insieme a una bulla recante l'impressione di sigillo del re ittita Tuthalija IV.

\subsection{Ipotesi pertinenti il cerimoniale officiato nell'edificio $C$}

Affinché non risulti pleonastico il tentativo di evidenziare i paralleli stilistici e funzionali fra gli oggetti votivi sopramenzionati, attraverso un'analisi comparativaassociativa, il risultato prodotto da questa correlazione appare essere circoscritto ad una sfera puramente congetturale. Ciò lo si evince soprattutto per l'assenza di elementi volti a rivelare la natura e la tipologia delle liturgie officiate nell'edificio C.

Fra le varie ipotesi formulate, la più attendibile risulta essere quella avanzata da $\mathrm{K}$. Bittel, e riproposta da F. Fischer, di vedere questa struttura quale camera sepolcrale, in relazione al ritrovamento nel vano 2 di resti ossei umani in prossimità di un pozzo per la raccolta dell'acqua piovana, coperto dal livellamento di epoca frigia ${ }^{34}$. La validità dell'interpretazione di C quale sacrario per delle liturgie ipoteticamente correlate al culto dei defunti si ravvisa anche e, soprattutto, in base alla presenza di una stele, recante un'iscrizione in geroglifici (fig. 10), di Tuthalija IV, che fu rinvenuta sopra l'apertura del pozzo, con l'epigrafe rivolta verso l'alto ${ }^{35}$. Quest'ultima, anticamente, doveva levarsi in alto come indicherebbe il paragone con un'analoga stele proveniente da Karahöyük, dove fu portata alla luce una lastra iscritta in situ alloggiata all'interno di una cavità in pietra ${ }^{36}$.

\section{CONCLUSIONI}

A dispetto degli elementi citati, sopra a tutti, l'iscrizione di Tuthalija IV, e i resti ossei in prossimità di una cisterna per l'acqua, che ha un'importanza fondamentale nelle celebrazioni liturgiche, la tesi proposta da K. Bittel di vedere in C una camera sepolcrale, necessita di dati ulteriori, tuttavia analogamente controverso appare essere lo schema congetturale stesso su cui si fonda l'identificazione di C quale camera sepolcrale. Nonostante, infatti, sia emersa una chiara interconnessione tra il complesso C a Büyükkale e il tempio di İnandiktepe, proprio grazie alla comparazione delle classi di materiali

31 La fossa risulta essere stata scavata in una fase immediatamente successiva la distruzione del tempio, come indica il taglio del pavimento nel vano 29, e pure dagli arredi cultuali asportati dalla cella del tempio e gettati al suo interno, fra cui si citano una coppia di tori in argilla, di cui paralleli furono rinvenuti, parimenti, nel vano M/19 dell'edificio M a Büyükkale (Özgüç 1988, 74, 111-112).

32 Presumibilmente trattasi dell'estremità superiore di uno scettro oppure di una spada.

33 Boehmer 1972, tav. XCII:2277.

34 Trattasi dei frammenti di un cranio e di un femore; Bittel 1935, 19; Fischer 1963, 38.

35 Bittel 1935, 19.

36 Özgüç - Özgüç 1949, 44. 
analizzate, in assenza di ulteriori elementi, non è possibile formulare ulteriori ipotesi al riguardo. È tuttavia importante rilevare che la continuità stilistica della varietà di brocca toreutica con versatoio e terminazione ad uncino, dall'epoca paleo-ittita all'età imperiale, indichi il persistere di una liturgia che ne prevedeva l'uso in atti libatori.

Una delle problematiche principali, considerata l'assenza di testimonianze testuali ed archeologiche, è legata proprio alla definizione del tipo di cerimoniale religioso officiato e, in aggiunta, se si tratta dello stesso rito celebrato sul vaso di İnandiktepe.

In relazione a ciò le differenze evidenziate fra le due scene che rappresentano l'atto libatorio (rispettivamente, la libagione compiuta dal sacerdote verso una divinità seduta su una sedia pieghevole fronte ad un altare, sul vaso di İnandiktepe, e la figura del re ittita, sull'ortostato da Alaca Höyük, che liba lui stesso ad una divinità alla testa di una processione) potrebbero indicare due riti diversi tra loro. Per quanto poi la presenza di resti ossei umani, in un ambiente, il N. 3, la cui contestualizzazione stratigrafica fu disturbata da posteriori attività in epoca frigia, apra il campo a congetture di vario tipo, l'ipotesi di una camera sepolcrale non risulta essere condivisibile, proprio alla luce del confronto con gli arredi cultuali rinvenuti nel tempio consacrato al dio della Tempesta a İnandıktepe. Le ragioni addotte per l'identificazione di C quale santuario si motivano, infatti, nell'associazione stilistica e funzionale con la classe degli arredi cultuali repertati nel tempio di İnandıktepe. Sebbene questa stessa correlazione non indichi una liturgia uniforme, gli elementi raccolti paiono comunque confutare una codifica per B-C-H legata al culto dei defunti.

\section{BIBLIOGRAFIA}

BITTEL, K.

1935 Vorläufiger Bericht über die Ausgrabungen in Boğazköy 1934: Mitteilungen der Deutsch Oriental Gesellschaft 73 (1935), pp. 13-28.

1937 Vorläufiger Bericht über die Ausgrabungen in Boğazköy 1936: Mitteilungen der Deutsch Oriental Gesellschaft 75 (1937), pp. 1-26.

BOEHMER, R.M.

1972 Die Kleinfunde von Boğazköy, aus den Grabungskampagnen 1931-1939 und 1952-1969 (Wissenschaftliche Veröffentlichung der Deutschen Orient-Gesellschaft 87), Berlin 1972.

FISCHER, F.

1963 Die Hetitische Keramik von Boğazköy (Wissenschaftliche Veröffentlichung der Deutschen Orient-Gesellschaft IV, 75), Berlin 1963, pp. 36-41.

NEVE, P.

1982 Büyükkale. Die Bauwerke. Grabungen 1954-1966, Berlin 1982.

ÖzGÜÇ, T.

1988 Inandıktepe. Eski Hitit çağında önemli bir kült merkezi/An important cult center in the Old Hittite Period (Türk Tarih Kurumu Basımevi V. 43), Ankara 1988, pp. 69-76; 78-80; 84-116.

ÖzGÜÇ, T. - ÖzGÜÇ, N.

1949 Karahöyük hafriyatı raporu /Ausgrabungen Von Karahoyük (Türk Tarih Kurumu Yayınları V.7), Ankara 1949. 
YILDIRIM, T.

2009 Hüseyindede: A settlement in northern-central Anatolia. Contributions to Old Hittite Art: F. PeCchioli DAdDi - G. ToRri - C. CoRTi (eds.), New Perspectives in light of recentresearch. Acts of the international conference held at the University of Florence (Studia Asiana 5), Firenze 2009, pp. 235-246. 


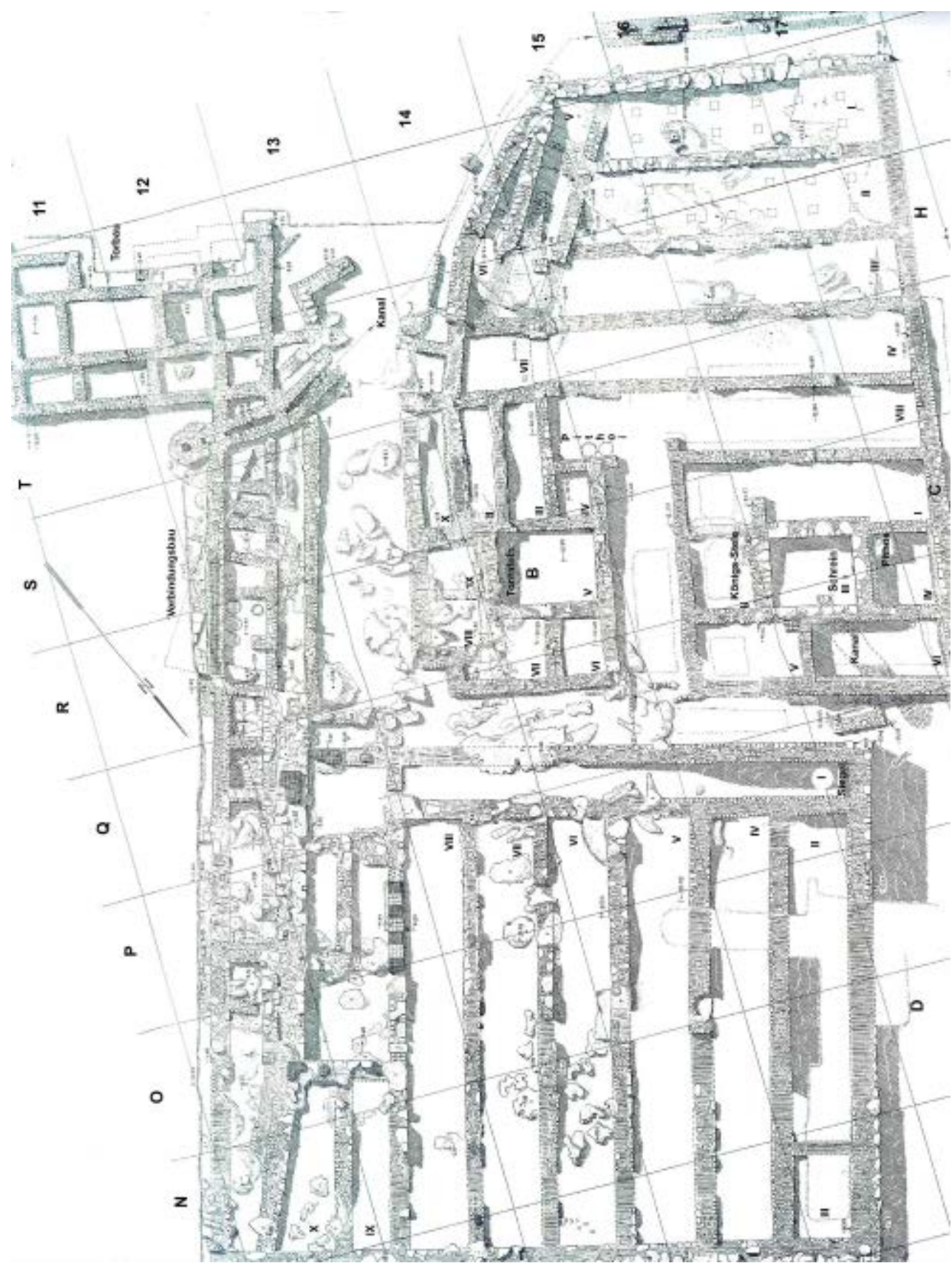

Fig. 1 - Pianta complesso edifici B-C-H (Neve 1982, pianta 38). 


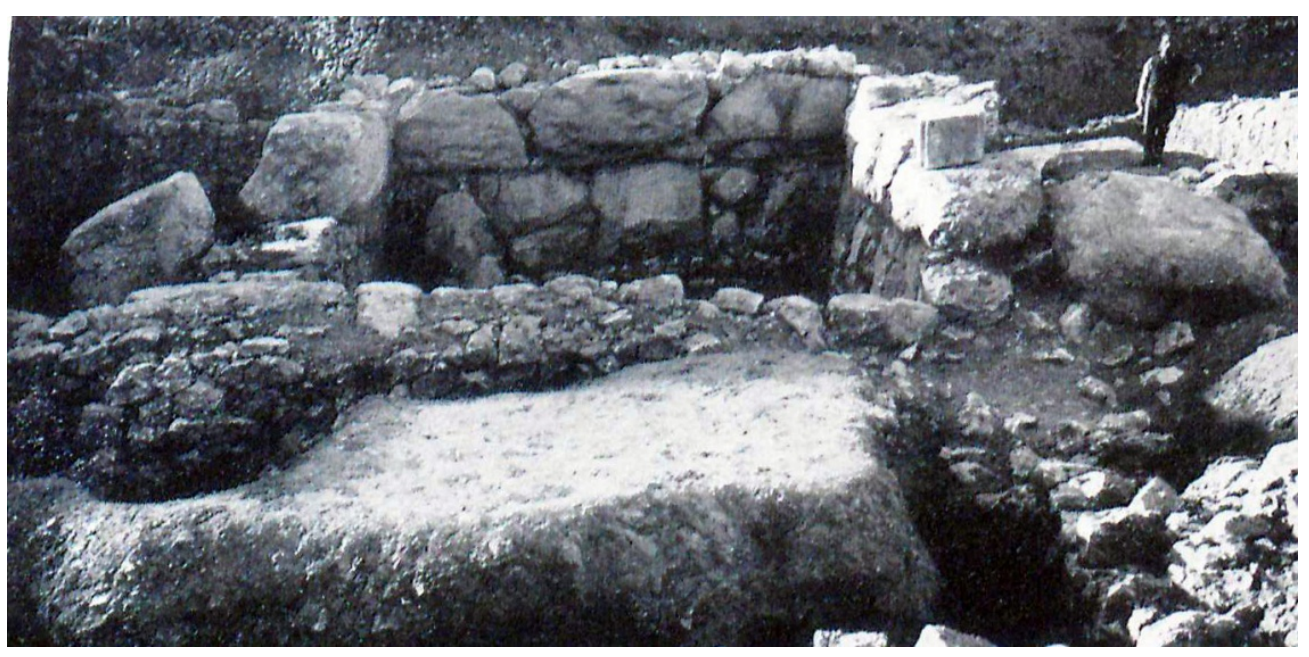

Fig. 2 - Vano III o ‘Schrein’ da nord-est (Bittel 1937, fig. 9).
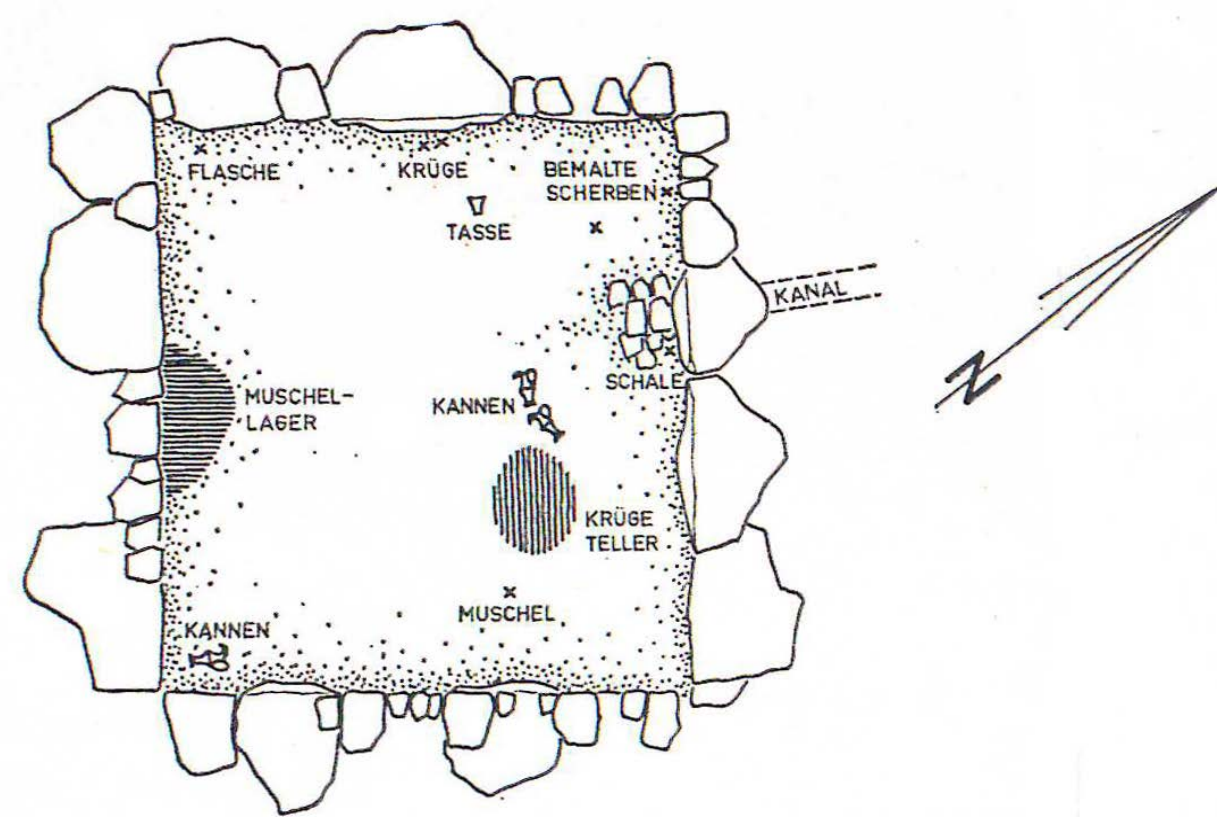

Fig. 3 - Distribuzione dei ‘Votivegefasse’ nel Vano III o ‘Schrein’ (Bittel 1937, fig. 12). 

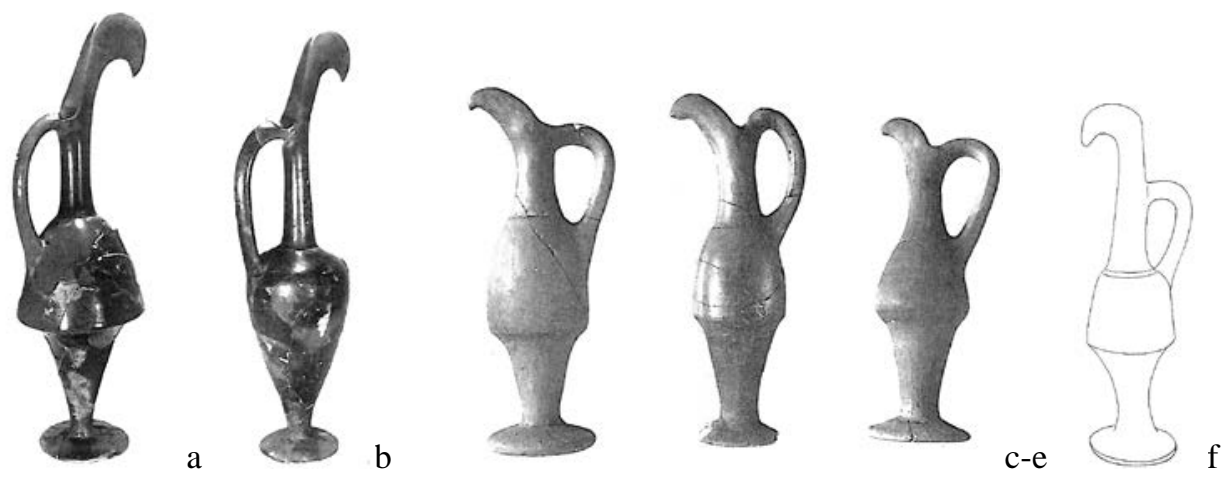

Fig. 4 - Brocche con versatoio con terminazione a rampone: a-b, dal vano 2 del tempio di İnandıktepe (Özgüç 1988, tav. 23:1-2); c-e, dal vano 3 dell'edificio C (Fischer 1963, tav. 29:260-262); f, da Alaca Höyük ‘Niveau hittite postérieur’ (Fischer 1963, fig. 4:10).

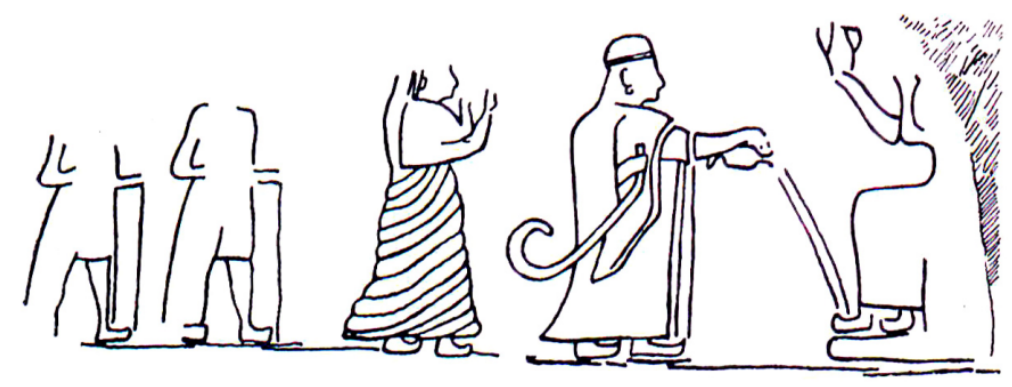

Fig. 5 - Ortostato con rilievo raffigurante scena di libagione con brocca di tipo toreutico da Alaca Höyük (Bittel 1935, fig. 11).

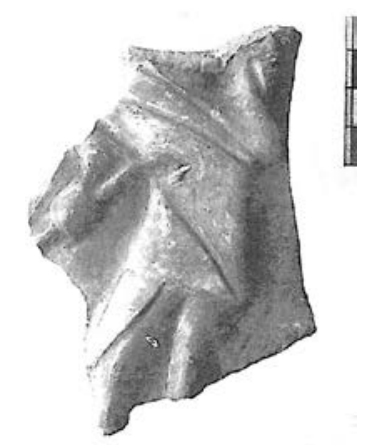

Fig. 6 - Frammento di vaso con rilievo da Karahöyük/elbistan (Özgüç 1949, tav. XLVII:2). 


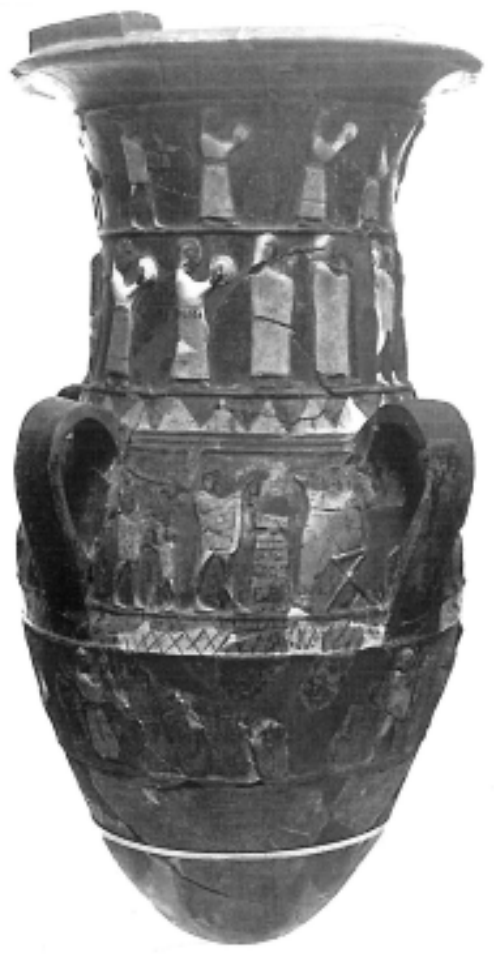

a
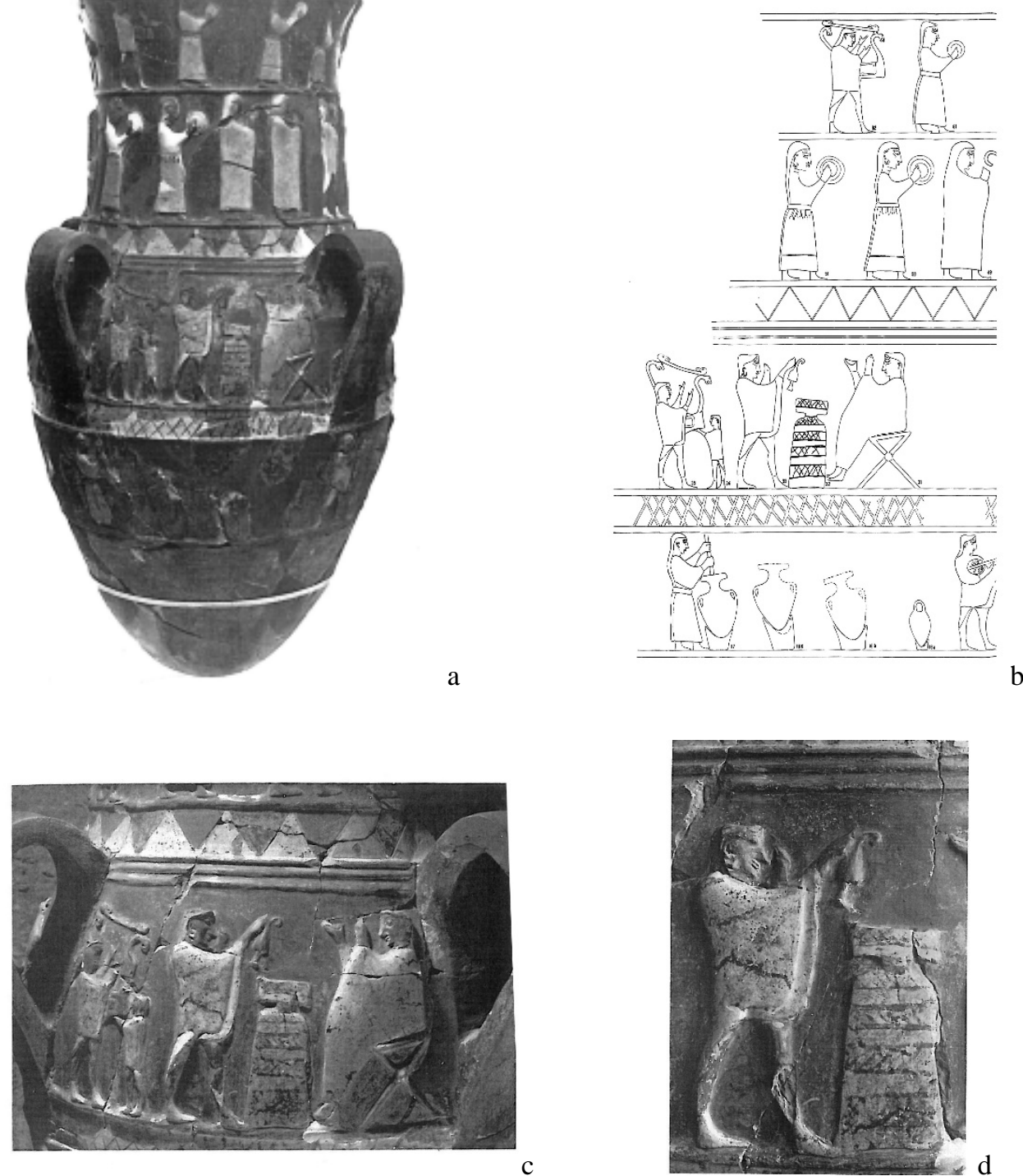

Fig. 7 - Vaso con rilievi a fasce dal tempio di İnandıktepe (Özgüç 1988, tav. 40) e particolari della scena libatoria con brocca con terminazione ad uncino nel secondo registro (Özgüç 1988, fig. 64, tavv. 57:1; 54:1). 

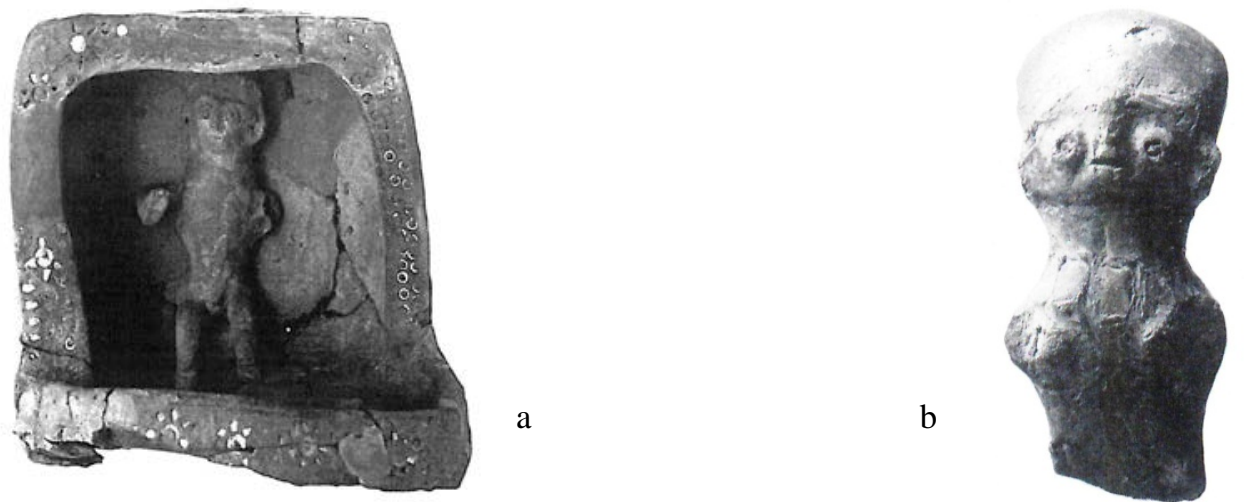

Fig. 8 - Figure fittili maschili di divinità: a, dal pozzo di scarico nel vano 29 del tempio di İnandıktepe (Özgüç 1988, tav. 63:1a); b, dall'edificio C (Bittel 1937, fig. 13, a).

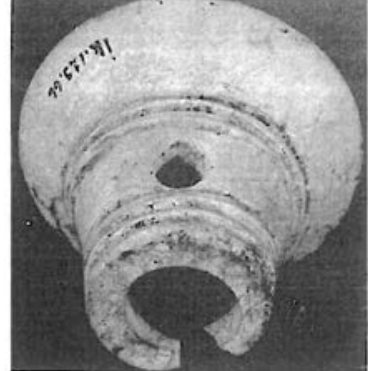

a

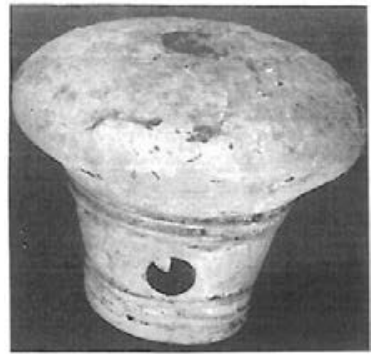

$\mathrm{b}$

Fig. 9 - Pomello o impugnatura in calcare dal pozzo di scarico nel vano 29 del tempio di İnandiktepe (Özgüç 1988, tav. 84:1a-b).

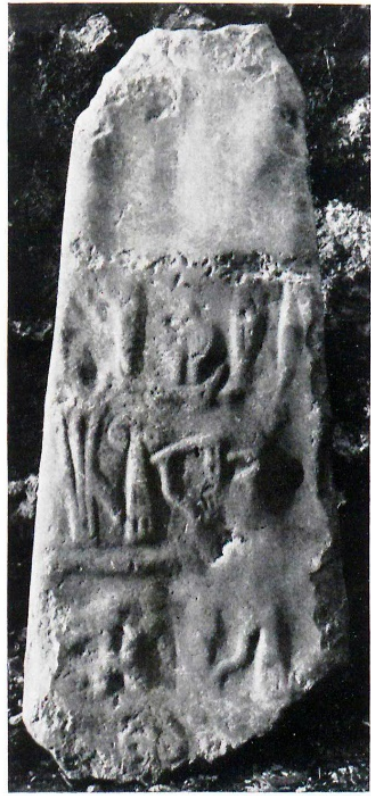

Fig. 10 - Stele con iscrizione in geroglifico di Tuthalija IV dal vano 2 dell'edificio C (Bittel 1935, fig. 21). 


\section{SOMMARIO}

DipartimENTo SCIENZE DELl'ANTICHITÀ

SEZIONE di ORIENTALISTICA

\section{VICINO ORIENTE \\ XIX - 2015}

L. Nigro - Bethlehem in the Bronze and lron Ages
in the light of recent discoveries by the Palestinian MOTA-DACH

$\checkmark$. Pisaniello - Parallel passages among Hittite-Luwian rituals:

for the restoration of $K U B 35.146$

F. Spagnoli - Una testa di sileno in bronzo da Mozia

N. Chiaren

dall Area sacra del Kothon a Mozia

G. Labisi - al-Fudayn: an Umayyad residence in Northern Jordan

P. Buzi - Early Christianity in the Fayyūm: the new contribution of archaeology

I. Materia - Preliminary notes on the ware depicted on the ceiling

of the Cappella Palatina in Palermo

S. Autiero - Indian Ocean trade:

a reassessment of the pottery find
$\left(3^{\text {rd }}\right.$ century $B C-S^{S^{t h}}$ century $\left.A D\right)$

M.M. Jamhawi - N. Al-Shakarchi - I. Al-Hashimi

Assessment of tourists' satisfaction in the downtown of Amman

SCAVI E RICERCHE

L. Nigro - C. Fiaccavento - M. Jaradat - J. Yasine

A

L. Nigro - D. Montanari - M. Ghayyada - J. Yasine

A Middle Bronze and Iron Age necropolis near Bethlehem (Palestine)

L. Nigro - G. Ripepi - I. Hamdan - J. Yasine

15 Interim Report

and valorization of archaeological heritage

R. Francia - L'archivio di tavolette del complesso B-C-H di Büyükkale

organizazione degli archivi reali ittiti. Considerazioni preliminari

V. Pisaniello - La collezione di tavolette del complesso B-C-H di Büyükkale

T. De Vincenzi - L'archivio di tavolette del complesso B-C-H
sull'acropoli di Büyükkale

Museo del Vicino Oriente, Egitto e Mediterraneo

L. Nigro - Il nuovo allestimento del Museo del Vicino Oriente,

Egitto e Mediterraneo della Sapienza

D. Montanari - Bollettino delle attività del Museo del Vicino Oriente,

Egito e Meditraneo della Sapienza, anno 2015

RECENSIONI

A. Orsingher - E. PAPPA (2013), Early Iron Age Exchange in the West:

(Ancient Near Eastern Studies Supplement Series 43)

Leuven - Paris - Walpole 2013, MA.: Peeters $\frac{1}{2}$

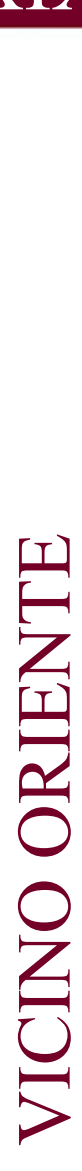

345

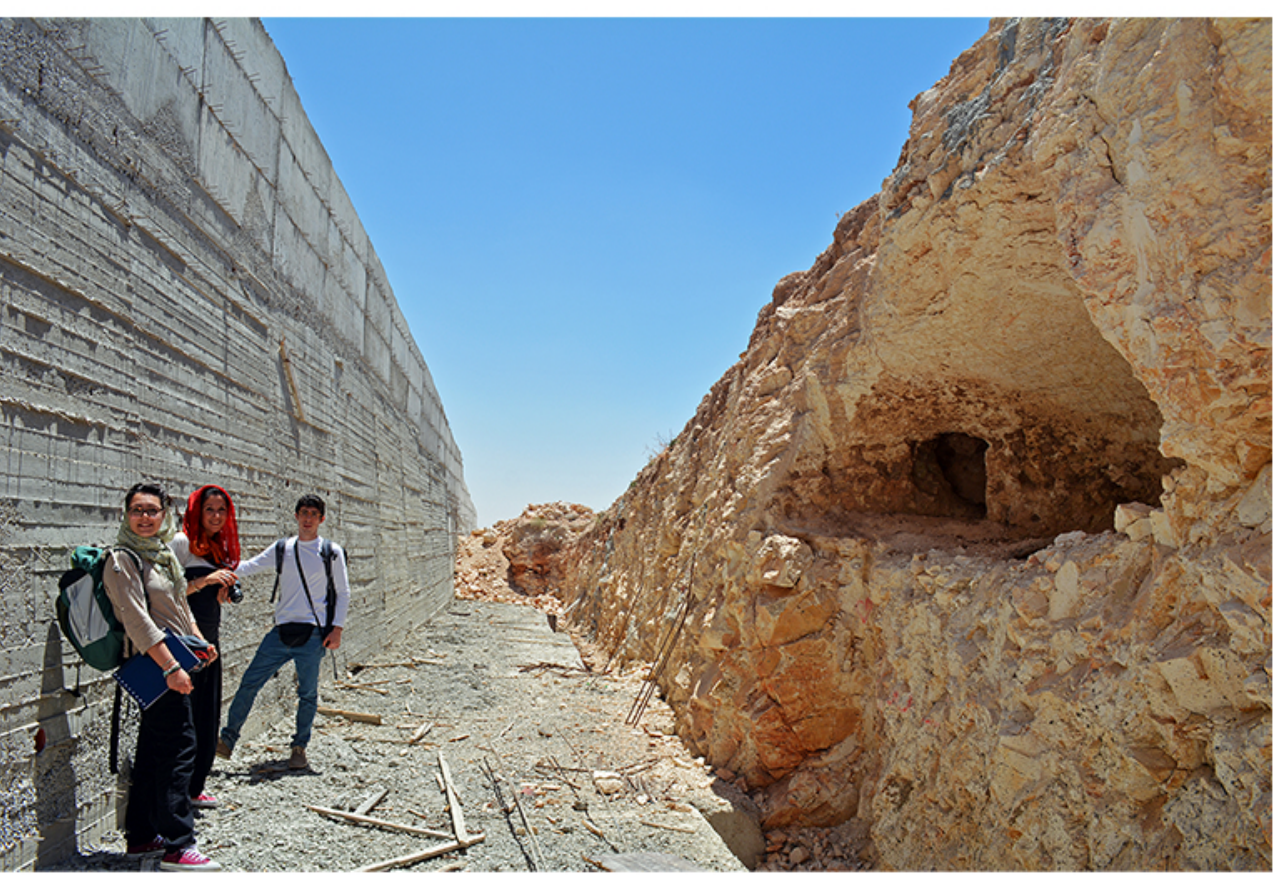

ROMA 2015 


\section{VICINO ORIENTE XIX - 2015}


VICINO ORIENTE

SAPIENZA UNIVERSITÀ DI ROMA

DIPARTIMENTO SCIENZE DELL'ANTICHITÀ

SEZIONE DI ORIENTALISTICA

Scientific Editor: Lorenzo Nigro

International Scientific Committee: Brian Rose, Frank Braemer, Mounir Fantar, Piero Bartoloni, Thomas Schaefer, Zeidan Kafafi

National Scientific Committee: Carlo Giovanni Cereti, Maria Vittoria Fontana, Sebastiano Tusa, Massimiliano Marazzi

Editorial Board: Daria Montanari, Chiara Fiaccavento

Tipografia: SK7 - Roma

ISSN 0393-0300

Rivista con comitato di referee

Journal with international referee system

www.lasapienzatojericho.it/SitoRivista/Journal/Rivista.php

In copertina: Tomba B9, necropoli di Khalet al-Jam’a (Betlemme). 
VICINO ORIENTE

SAPIENZA UNIVERSITÀ DI ROMA

DIPARTIMENTO SCIENZE DELL'ANTICHITÀ

SEZIONE DI ORIENTALISTICA

\section{SOMMARIO}

ARTICOLI

L. Nigro - Bethlehem in the Bronze and Iron Ages

in the light of recent discoveries by the Palestinian MOTA-DACH

V. Pisaniello - Parallel passages among Hittite-Luwian rituals:

for the restoration of KUB 35.146

F. Spagnoli - Una testa di sileno in bronzo da Mozia

N. Chiarenza - Una matrice per terrecotte con sileno dall'Area sacra del Kothon a Mozia

G. Labisi - al-Fudayn: an Umayyad residence in Northern Jordan

P. Buzi - Early Christianity in the Fayyūm: the new contribution of archaeology

I. Materia - Preliminary notes on the ware depicted on the ceiling

of the Cappella Palatina in Palermo

S. Autiero - Indian Ocean trade:

a reassessment of the pottery finds from a multidisciplinary point of view ( $3^{\text {rd }}$ century $B C-5^{\text {th }}$ century $\left.A D\right)$

M.M. Jamhawi - N. Al-Shakarchi - I. Al-Hashimi

Assessment of tourists' satisfaction in the downtown of Amman

SCAVI E RICERCHE

L. Nigro - C. Fiaccavento - M. Jaradat - J. Yasine Archaeology from A to Z: Abu Zarad, an ancient town in the heartland of Palestine

L. Nigro - D. Montanari - M. Ghayyada - J. Yasine

Khalet al-Jam'a. A Middle Bronze and Iron Age necropolis near Bethlehem (Palestine) 185 
VICINO ORIENTE

SAPIENZA UNIVERSITÀ DI ROMA

DIPARTIMENTO SCIENZE DELL'ANTICHITÀ

SEZIONE DI ORIENTALISTICA

L. Nigro - G. Ripepi - I. Hamdan - J. Yasine

The Jericho Oasis Archaeological Park - 2015 Interim Report.

Italian-Palestinian Cooperation for protection

and valorization of archaeological heritage

R. Francia - L'archivio di tavolette del complesso B-C-H di Büyükkale

e l'organizzazione degli archivi reali ittiti. Considerazioni preliminari

V. Pisaniello - La collezione di tavolette del complesso B-C-H di Büyükkale

T. De Vincenzi - L'archivio di tavolette del complesso B-C-H sull'acropoli di Büyükkale

Museo del Vicino ORIente, Egitto e MediterRaneo

L. Nigro - Il nuovo allestimento del Museo del Vicino Oriente,

Egitto e Mediterraneo della Sapienza

D. Montanari - Bollettino delle attività del Museo del Vicino Oriente,

Egitto e Mediterraneo della Sapienza, anno 2015

\section{RECENSIONI}

A. Orsingher - E. PAPPA (2013), Early Iron Age Exchange in the West:

Phoenicians in the Mediterranean and the Atlantic

(Ancient Near Eastern Studies Supplement Series 43),

Leuven - Paris - Walpole 2013, MA.: Peeters 